\title{
Aza-deoxycytidine induces apoptosis or differentiation via DNMT3B and targets embryonal carcinoma cells but not their differentiated derivatives
}

\author{
P Wongtrakoongate ${ }^{*, 1,2}, \mathrm{~J} \mathrm{Li}^{1,3}$ and P W Andrews ${ }^{1}$ \\ ${ }^{1}$ Centre for Stem Cell Biology, University of Sheffield, Sheffield S10 2TN, UK
}

Background: Teratocarcinoma is a malignant male germ cell tumour, which contains stem cells and differentiated cancer tissues. DNMT3B has been shown to be highly expressed in human teratocarcinoma stem cells, and to mediate cytotoxicity of Azadeoxycytidine (Aza-dC) in a pluripotent stem cell line NTERA2.

Methods: We have established DNMT3B or POU5F1 (hereafter referred to as OCT4) knockdown in teratocarcinoma stem cells N2102Ep and TERA1 and in the pluripotent NTERA2 by a doxycycline-inducible system, and tested the cytotoxicity induced by Aza-dC.

Results: Silencing of DNMT3B led to apoptosis of human teratocarcinoma stem cells N2102Ep and TERA1. Further, we found that induction of apoptosis or differentiation in NTERA2 and human embryonic stem cells by Aza-dC requires DNMT3B. To test whether Aza-dC inhibits proliferation of differentiated teratocarcinoma cells, we depleted OCT4 expression in N2102Ep and TERA1 cells treated with Aza-dC. Treatment with Aza-dC reduced cell number of differentiated cells to a lesser extent than their undifferentiated parental stem cells. Moreover, in contrast to the stem cells, Aza-dC failed to induce apoptosis of differentiated cells.

Conclusions: Our finding suggests that DNMT3B acts as an antiapoptotic gene in teratocarcinoma stem cells, and mediates apoptosis and differentiation of human pluripotent stem cells induced by Aza-dC, and that Aza-dC specifically induces apoptosis of teratocarcinoma stem cells.

Teratocarcinoma is one form of testicular germ cell tumour (GCT), the most common type of cancer occurring in young men between 20 and 40 years of age (Oosterhuis and Looijenga, 2005). These tumours contain both embryonal carcinoma (EC) cells, which represent the stem cells of the cancer, and a heterogeneous mixture of their differentiated derivatives. However, in some cases, these tumours may be composed entirely of EC cells, without any differentiated cell components, whereas many cell lines such as N2102Ep and TERA1 derived from testicular GCTs also appear to be composed of EC cells that have lost the ability to differentiate.
Such EC cells are termed 'nullipotent' and fail to differentiate under normal circumstances such as when grown as xenograft tumours or induced with agents like retinoic acid (Andrews et al, 1980), which do induce the differentiation of pluripotent EC cell line NTERA2 (Andrews, 1984; Andrews et al, 1984). The appearance of nullipotent EC cells may reflect selection of more aggressive variants during cancer progression. Nevertheless, nullipotent EC cells can be induced to differentiate following genetic manipulation to knockdown expression of OCT4 (Matin et al, 2004; Andrews et al, 2005).

\footnotetext{
*Correspondence: Dr P Wongtrakoongate; E-mail: p.wongtrakoongate@gmail.com

${ }^{2}$ Current address: Laboratory of Molecular Biology, National Institute of Diabetes and Digestive and Kidney Diseases, National Institutes of Health, Bethesda, Maryland 20892, USA

${ }^{3}$ Current address: R\&D, Bio-Research Tools, Life Science, GE Healthcare, Building CD1, The Maynard Centre, Forest Farm, Whitchurch, Cardiff CF14 7YT, Wales, UK
}

Received 17 December 2013; revised 13 February 2014; accepted 14 February 2014; published online 6 March 2014 
The cellular origin of GCT has been suggested by Skakkebaek and colleagues (Skakkebaek, 1972; Rajpert-De Meyts et al, 1998) to be carcinoma in situ (CIS), also known as intratubular germ-cell neoplasia unclassified lesion or testicular intratubular neoplasia. In addition, Rajpert-de Meyts and Hoei-Hansen (Rajpert-de Meyts and Hoei-Hansen, 2007) have proposed a hypothesis suggesting that these CIS cells are defective arrested primordial germ cells (PGCs) or gonocytes due to testicular dysgenesis. A transcriptomic analysis of CIS, early germ cells and several types of GCTs has indicated that CIS cells in fact resemble to PGCs/gonocytes (Sonne et al, 2009). The molecular similarity between CIS and gonocytes is further supported by low levels of DNA methylation and histone H3 lysine 9 dimethylation in CIS, which are consistent with low levels of those epigenetic marks in PGCs (Almstrup et al, 2010). An activation of embryonic programme similar to embryonic stem (ES) cells in CIS might lead to the development of teratocarcinoma stem cells (Almstrup et al, 2004; Skotheim et al, 2005; Kristensen et al, 2008; Alagaratnam et al, 2012).

Tumour eradication of teratocarcinoma can be achieved by treatment of those cancers with irradiation and cisplatin-based compounds, as they are sensitive to these therapies. The sensitivity of teratocarcinoma to radiation and DNA-damaging agents can be explained by their cell origin of the tumours, that is, CIS. As these agents are very toxic to embryonic cells including PGCs, CISderived cancer cells are therefore susceptible to these treatments. Thus, the loss of a stem cell phenotype of EC cells as they differentiate to form teratomas will result in a resistance of the cancers to radiation and chemotherapy (Oosterhuis and Looijenga, 2005). More than $70 \%$ of patients with teratocarcinoma can be cured by using cisplatin-based chemotherapy in combination with surgery. Nevertheless, the rest of patients cannot be cured due to an incomplete response or relapse. Moreover, testicular GCT is still a fatal cancer disease in approximately $5 \%$ of the patients. One potential chemotherapy to improve the treatment outcome is by combining cisplatin-based therapy with a DNA methylation inhibitor Aza-deoxycytidine (Aza-dC; Beyrouthy et al, 2009), which has been clinically used for treatment of patients with myelodysplastic syndrome and some solid tumours (Yang et al, 2010), and possesses an ability to inhibit proliferation of cancer stem-like cells (Tsai et al, 2012).

A number of gene expression analyses have found that DNMT3B, which encodes a de novo DNA methyltransferase, is highly expressed in nulipotent human EC cells at a level similar to the pluripotent EC cell line, NTERA2, and human ES cells (Sperger et al, 2003; Skotheim et al, 2005; Alagaratnam et al, 2012). In the present study, we found that silencing of DNMT3B induced apoptosis of nullipotent EC cells, N2102Ep and TERA1. However, DNMT3B knockdown did not induce apoptosis in pluripotent NTERA2 and ES cells, but did attenuate apoptosis or differentiation induced by Aza-dC in NTERA2 and ES cells, suggesting that DNMT3B is required for apoptosis or differentiation induced by Aza-dC. However, when N2102Ep and TERA1 were caused to differentiate by a knockdown of POU5F1 (hereafter referred to as OCT4) Aza-dC-induced apoptosis was antagonised. Our studies suggest differential roles of DNMT3B in nullipotent and pluripotent stem cells, and highlight an evidence of cancer stem cells, but not their differentiated derivatives, which undergo apoptosis induced by the epigenetic drug.

\section{MATERIALS AND METHODS}

Cell culture. Human teratocarcinoma stem cells N2102Ep and TERA1 (Andrews et al, 1980) and NTERA2 (Andrews et al, 1984) were grown using DMEM (high glucose, no pyruvate) (PAA) supplemented with $10 \%$ fetal calf serum (FCS; Hyclone, Rockford,
IL, USA). N2102Ep and TERA1 were passaged by $0.25 \%$ trypsin/ EDTA at day 5 of culture as previously described (Andrews et al, 1980), whereas NTERA2 were passaged at $1: 3$ ratio by using glass beads. The human ES cell line $\mathrm{H} 7$ (Thomson et al, 1998) was grown in knockout-DMEM supplemented with 20\% knockoutserum replacement, $1 \mathrm{X}$ non-essential amino acids, $1 \mathrm{~mm}$ glutamine, $0.1 \mathrm{~mm}$ beta-mercaptoethanol and $4 \mathrm{ng} \mathrm{ml}^{-1} \mathrm{bFGF}$ (Invitrogen, Grand Island, NY, USA) seeded on $6 \times 10^{3}$ cells $\mathrm{cm}^{-2}$ mitomycin C-treated MF-1 mouse embryonic fibroblasts, and placed at $37^{\circ} \mathrm{C}$ under a humidified atmosphere of $5 \% \mathrm{CO}_{2}$ incubator. Cells were passaged every 5-7 days using collagenase type IV (Invitrogen) and scraped with glass beads (Sigma, St Louis, MO, USA). For Aza-dC treatment, cells were grown in medium supplemented with $10 \mathrm{~nm}$ Aza-dC (Sigma); cultures were maintained for 7 days during which the culture medium was changed every other day.

Establishment of shRNAi-inducible cell lines. Tetracycline repressor (TetR)-overexpressing cell lines N2102Ep, TERA1 and NTERA2 were established by transfecting $1 \times 10^{6}$ cells with $5 \mu \mathrm{g}$ pCAG-TetRnls-IRES-Puromycin (Zafarana et al, 2009). Transfectants were seeded on $90 \mathrm{~mm}^{2}$ cell culture dishes. Puromycinresistant colonies were selected using $3 \mu \mathrm{g} \mathrm{ml}^{-1}$ puromycin (Sigma). Stable TetR-over-expressing N2102Ep, TERA1 and NTERA2 cell lines were subsequently transfected with $5 \mu \mathrm{g}$ pSuperior-Neo (Oligoengine, Seattle, WA, USA) containing siRNA targeting DNMT3B (Wongtrakoongate et al, 2014). N2102Ep and TERA1 cell lines harbouring OCT4 shRNAi construct were also established using the previously reported OCT4 target sequence (Zafarana et al, 2009). Stable transfected stem cell colonies were selected using $750 \mu \mathrm{g} \mathrm{ml}^{-1} \mathrm{G} 418$ (Invitrogen). Reverse transcription quantitative polymerase chain reaction and western blotting were employed to validate expression of knockdown-target genes in cells grown with or without $1 \mu \mathrm{g} \mathrm{ml}^{-1}$ doxycycline (Dox).

Cloning-efficiency assay. Cells were washed once with PBS. N2102Ep and TERA1 were disaggregated to a single-cell level using $0.25 \%$ trypsin, whereas $0.05 \%$ trypsin was used for NTERA2 and human ES cells H7. Cells were plated at 250 cells (for human teratocarcinoma stem cells) or at 10000 cells (for human ES cells) in six-well plates. After a 2-week culture, cells were washed once with PBS and ice-cold methanol, respectively. Cells were fixed with $1 \mathrm{ml}$ of the methanol for $15 \mathrm{~min}$ at room temperature. The methanol was removed, and cells were incubated with $1 \mathrm{ml}$ of a crystal violet solution $(0.4 \%(\mathrm{w} / \mathrm{v})$ in methanol) for another $15 \mathrm{~min}$. Unstained crystal violet was washed off by tap water. The plates were dried and were counted for colony numbers.

Flow cytometry. Flow cytometry was performed as previously described (Draper et al, 2002). Briefly, cultured cells were harvested by trypsinisation. One hundred thousand cells in $100 \mu \mathrm{l}$ PBS with $10 \%$ FCS were treated with MC631 monoclonal antibody recognising the SSEA3 antigen (Shevinsky et al, 1982) at $1: 10$ dilution for $15 \mathrm{~min}$. Cells were then washed and were incubated with Cy5-conjugated anti-rat secondary antibody (Jackson Laboratory, West Grove, PA, USA) for $15 \mathrm{~min}$. Cells were washed with Annexin $\mathrm{V}$ binding buffer (Invitrogen), and were incubated with FITC-conjugated Annexin V for $30 \mathrm{~min}$ at room temperature. The samples were analysed with the Cyan flow cytometer (Dako Cytomation, Carpinteria, CA, USA).

Reverse transcription quantitative polymerase chain reaction. RNA was extracted using TRIzol reagent (Invitrogen). Complementary DNA synthesis was performed with $1 \mu \mathrm{g}$ RNA using SuperScript II Reverse Transcriptase (Invitrogen). Then, qPCR was carried on by using SYBR Green JumpStart Taq ReadyMix (Sigma) in a total volume of $20 \mu \mathrm{l}$ each well with an iCycler iQ system (Bio-Rad, Hercules, CA, USA). Primer sequences are available upon request. 
Western blotting. Cell pellets were resuspended in RIPA buffer and were subjected to sonification. Protein concentration was determined using a bicinchoninic acid protein assay kit (Thermo Scientific, Rockford, IL, USA). Each well was loaded with $20 \mu \mathrm{g}$ of proteins. Proteins were transferred to PVDF membrane (Bio-Rad). Antibodies for western blotting are as followed; DNMT3B (sc-20704) and OCT4 (sc-5279) from Santa Cruz (Dallas, TX, USA); ACTB (A5316) from Sigma.

\section{RESULTS}

DNMT3B is important for clonal propagation of EC cells. To ascertain whether DNMT3B might be important for survival of human EC cells, we have generated Dox-inducible DNMT3B knockdown in human teratocarcinoma stem cell lines N2102Ep and TERA1 (Andrews et al, 1980). In addition, we have also established the inducible DNMT3B knockdown using a pluripotent stem cell line NTERA2, which possesses a unique ability to differentiate by retinoic acid (Andrews, 1984). We show that the expression of DNMT3B was decreased upon induction of Dox (Figure 1A). The human ES cell line H7 harbouring the DNMT3B inducible knockdown cassette, which has been established previously (Wongtrakoongate et al, 2014), also downregulated expression of DNMT3B by Dox treatment (Figure 1A). This result indicates that DNMT3B expression level can be silenced by the Dox-inducible shRNAi.

Next, we performed cloning-efficiency assay to determine whether DNMT3B might facilitate clonal propagation of teratocarcinoma stem cells. The clonal ability test reveals that silencing of $D N M T 3 B$ led to a reduction of cloning efficiency of EC cells N2102Ep and TERA1 (Figure 1B), suggesting a role of DNMT3B

A
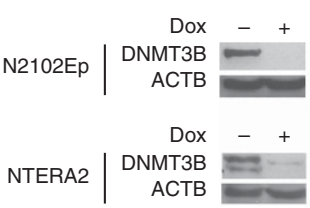

B
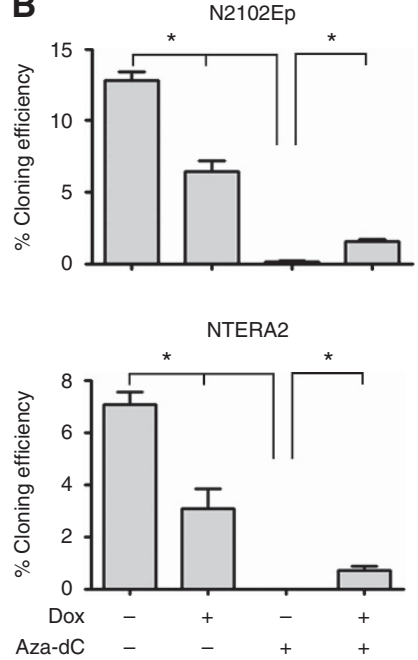
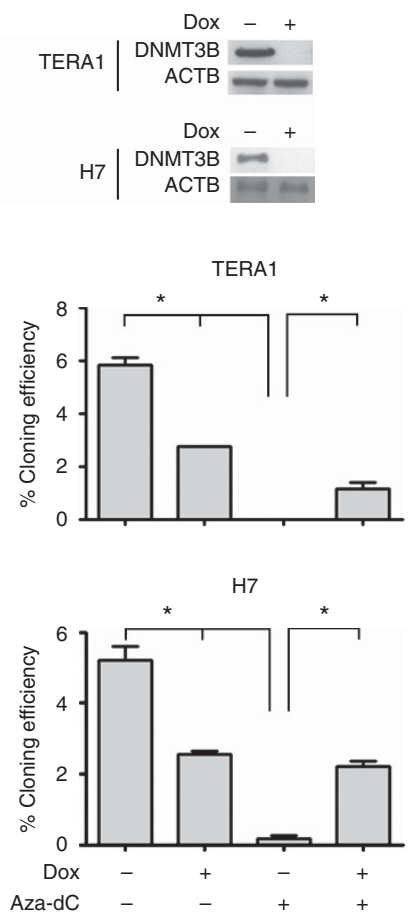

Figure 1. DNMT3B is not only important for clonal ability of stem cells, but also mediates a reduction of clonal ability induced by Aza-dC treatment. (A) Western blotting of DNMT3B in N2102Ep, TERA1, NTERA2 and H7 ES cells with doxycycline (Dox) treatment to knockdown DNMT3B. (B) Cloning efficiency was performed in N2102Ep, TERA1, NTERA2 and H7 cells with doxycycline (Dox) and/or Aza-dC treatment. Data are represented as mean \pm s.d.; $n=3$; $\star P<0.05$. in clonal propagation of the cancer stem cells. Similarly, DNMT3B knockdown also reduced clonal ability of human pluripotent stem cells NTERA2 and H7 (Figure 1B).

Aza-dC impairs clonal propagation via DNMT3B. DNMT has been proposed to mediate DNA mutagenicity and hence cellular cytotoxicity induced by Aza-dC through a covalent trapping mechanism between Aza-dC-incorporated DNA adduct and the methyltransferase (Juttermann et al, 1994; Jackson-Grusby et al, 1997). To define whether Aza-dC treatment might diminish colony survival, and whether DNMT3B might mediate the cytotoxic effect in human nullipotent EC cells, N2102Ep and TERA1, and human pluripotent stem cells NTERA2 and H7, DNMT3B expression was silenced for 3 days, and the cells were subsequently treated with Aza-dC. The result shows that Aza-dC treatment reduced cloning efficiency of the stem cells to a greater extent than the DNMT3B knockdown (Figure 1B). Upon Aza-dC treatment, we found that further downregulation of DNMT3B by shRNAi elevated colonyforming numbers in the stem cells, indicating that Aza-dC impedes survival of the cancer stem cells and pluripotent stem cells partly through a mechanism involving DNMT3B.

DNMT3B acts as an antiapoptotic gene in human EC cells. Next, apoptosis assay using a double staining of Annexin V together with the stem cell marker SSEA3 was employed to elucidate whether silencing of DNMT3B induces apoptosis of human nullipotent stem cells N2102Ep and TERA1 and pluripotent stem cells NTERA2 and H7. Upon DNMT3B silencing, population numbers of SSEA3 ${ }^{+} /$Annexin ${ }^{+}$, of which represents 'apoptotic stem cells', in Dox-treated N2102Ep and TERA1 were two-fold increased approximately in comparison with the controls (Figure $2 \mathrm{~A}$ and $\mathrm{B}$ ). On the other hand, the numbers of SSEA3 ${ }^{+} /$ Annexin $\mathrm{V}^{+}$population were not increased in the pluripotent stem cell lines NTERA2 and H7 ES cells (Figure 3A and B). These results suggest that DNMT3B might prevent apoptosis in the human nullipotent EC cells N2102Ep and TERA1, but not in pluripotent NTERA2 and human ES cells.

Aza-dC induces apoptosis of pluripotent stem cells NTERA2 through DNMT3B. We found that Aza-dC treatment led to an increase in the numbers of SSEA3 ${ }^{+} /$Annexin $\mathrm{V}^{+}$population in N2102Ep, TERA1, NTERA2 and H7 (Figure 2), suggesting that the apoptotic effect of Aza-dC on the stem cells is a general feature as in mouse ES cells (Juttermann et al, 1994; Oka et al, 2005). We also observed that in pluripotent NTERA2 cells treated with Aza-dC, a further depletion of DNMT3B by shRNAi resulted in a reduction in the SSEA3 ${ }^{+} /$Annexin $\mathrm{V}^{+}$population compared with cells treated with Aza-dC alone (Figure 3A). In contrast, the numbers of SSEA3 $^{+} /$Annexin $\mathrm{V}^{+}$population of N2102Ep, TERA1 and H7 treated with Aza-dC were similar between without or with DNMT3B silencing (Figure $2 \mathrm{~A}$ and $\mathrm{B}$ and Figure $3 \mathrm{~B}$ ). These results suggest that DNMT3B mediates an induction of apoptosis induced by Aza-dC in the pluripotent stem cells NTERA2 but not in N2102Ep, TERA1 and human ES cells.

Aza-dC induces differentiation of human ES cells through DNMT3B. As Aza-dC has been originally reported to induce cellular differentiation (Taylor and Jones, 1979; Jones and Taylor, 1980), we next asked whether Aza-dC might induce differentiation of human teratocarcinoma stem cells N2102Ep and TERA1 and pluripotent stem cells NTERA2 and H7. Treatment with Aza-dC resulted in an increase in numbers of SSEA3 ${ }^{-} /$Annexin $\mathrm{V}^{-}$cells, of which represents 'non-apoptotic differentiated cells', in pluripotent NTERA2 and H7 (Figure 3). On the other hand, the numbers of SSEA3 ${ }^{-}$/Annexin $\mathrm{V}^{-}$cells in N2102Ep and TERA1 were not increased by Aza-dC treatment (Figure 2A and B). This result indicates that Aza-dC induces differentiation of pluripotent NTERA2 and H7 cells. 
A

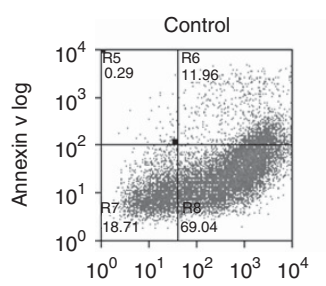

N2102Ep DNMT3B shRNAi

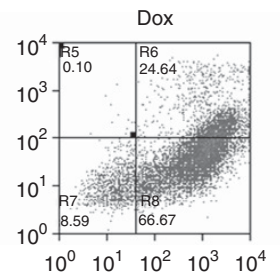

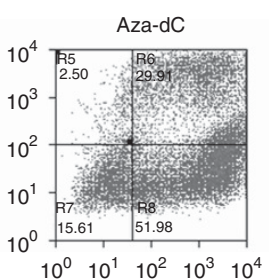
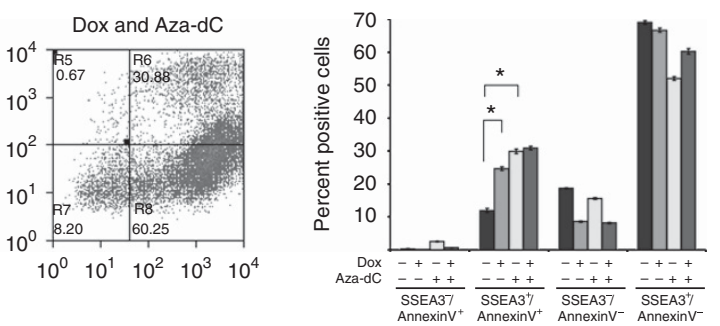

B
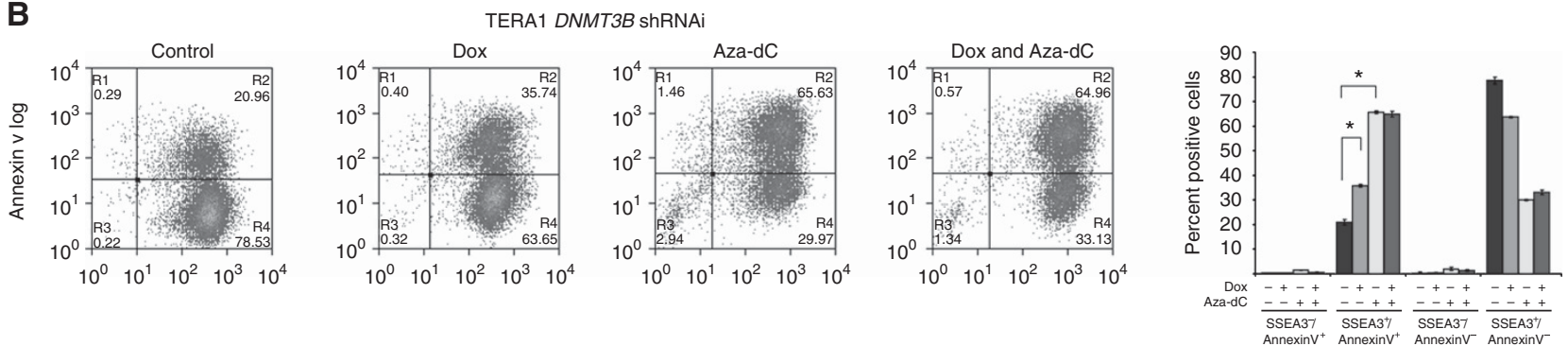

C
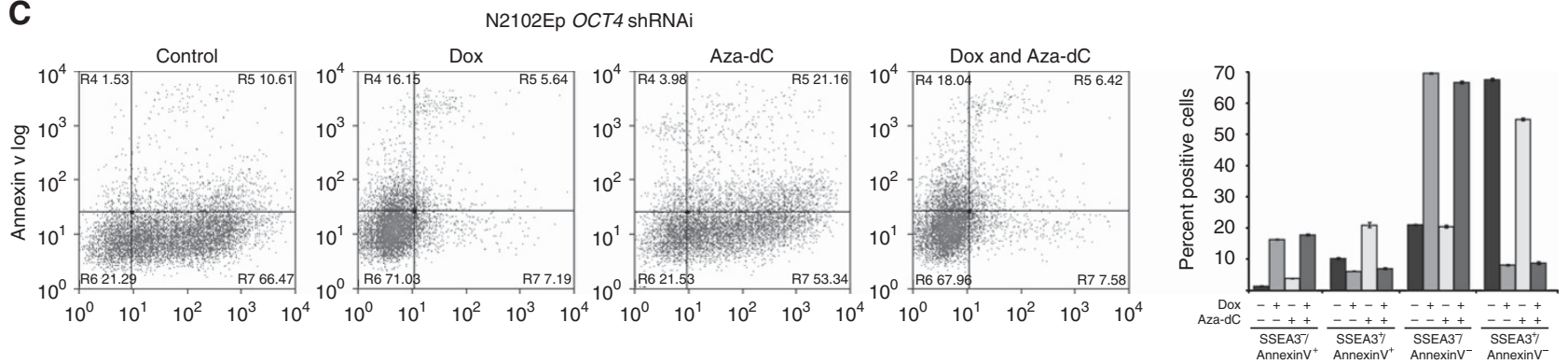

D
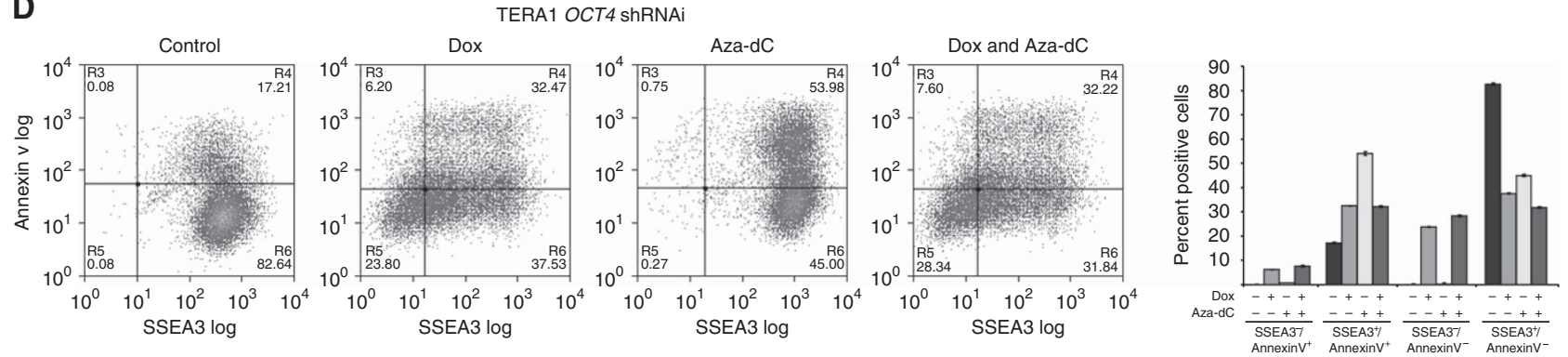

Figure 2. DNMT3B prevents apoptosis of nullipotent EC cells N2102Ep and TERA1. Flow cytometry analysis of DNMT3B knockdown in (A) N2102Ep and (B) TERA1, and OCT4 knockdown in (C) N2102Ep and (D) TERA1. Data are represented as mean \pm s.d.; $n=3 ;{ }^{\star} P<0.05$.

To establish whether DNMT3B mediates differentiation of pluripotent NTERA2 and H7 induced by Aza-dC, we treated the cells with Aza-dC in the absence or presence of DNMT3B silencing. In human ES cells $\mathrm{H} 7$ treated with Aza-dC, a further depletion of DNMT3B by shRNAi resulted in less cell number of SSEA3 ${ }^{-}$/ Annexin $\mathrm{V}^{-}$population than cells treated with Aza-dC only (Figure 3B). In contrast, the number of SSEA3 ${ }^{-} /$Annexin $^{-}$cells of NTERA2 treated with Aza-dC was similar between without or with DNMT3B silencing (Figure 3A). This result suggests that DNMT3B mediates an induction of differentiation induced by Aza$\mathrm{dC}$ in human ES cells but not in pluripotent stem cells NTERA2.

Consistent with flow cytometry analysis, we found that Aza-dC treatment led to a reduction of the expression level of stem cellassociated genes OCT4, NANOG and SOX2 in pluripotent NTERA2 and H7 ES cells (Figure 4). In contrast, Aza-dC treatment did not downregulate expression of the stem cell genes in the nullipotent EC cells N2102Ep and TERA1. Further, silencing of DNMT3B in AzadC-treated NTERA2 and H7 resulted in a higher expression of
OCT4, NANOG and SOX2 compared with cells treated with Aza-dC alone. This result indicates that DNMT3B might also mediate downregulation of OCT4, NANOG and SOX2 in pluripotent NTERA2 and H7 ES cells induced by Aza-dC.

Resistance of Aza-dC-induced apoptosis of differentiated teratocarcinoma stem cells. Although many human EC cell lines fail to differentiate after manipulation of culture conditions, we have previously shown that N2102Ep and TERA1 do differentiate following knockdown of OCT4 using siRNA (Matin et al, 2004; Andrews et al, 2005). To test whether these differentiated derivative cells undergo apoptosis by Aza-dC induction similar to that observed in their stem cell parents (Figure 2A and B), we have established shRNAi-inducible OCT4 knockdown cell lines of N2102Ep and TERA1 (Figure 5A). Consistent with the previous works, the inducible OCT4 knockdown N2102Ep and TERA1 by shRNAi showed an upregulation of genes representing trophoblastic and endodermal lineages, indicating their differentiation (Figure 5B). 
A
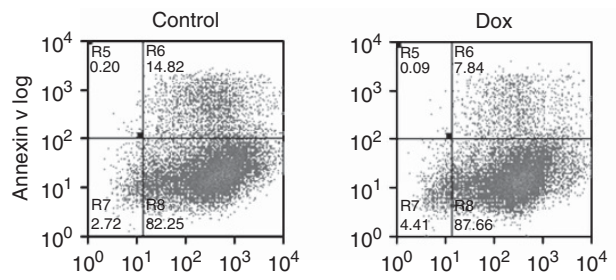

NTERA2 DNMT3B ShRNAi
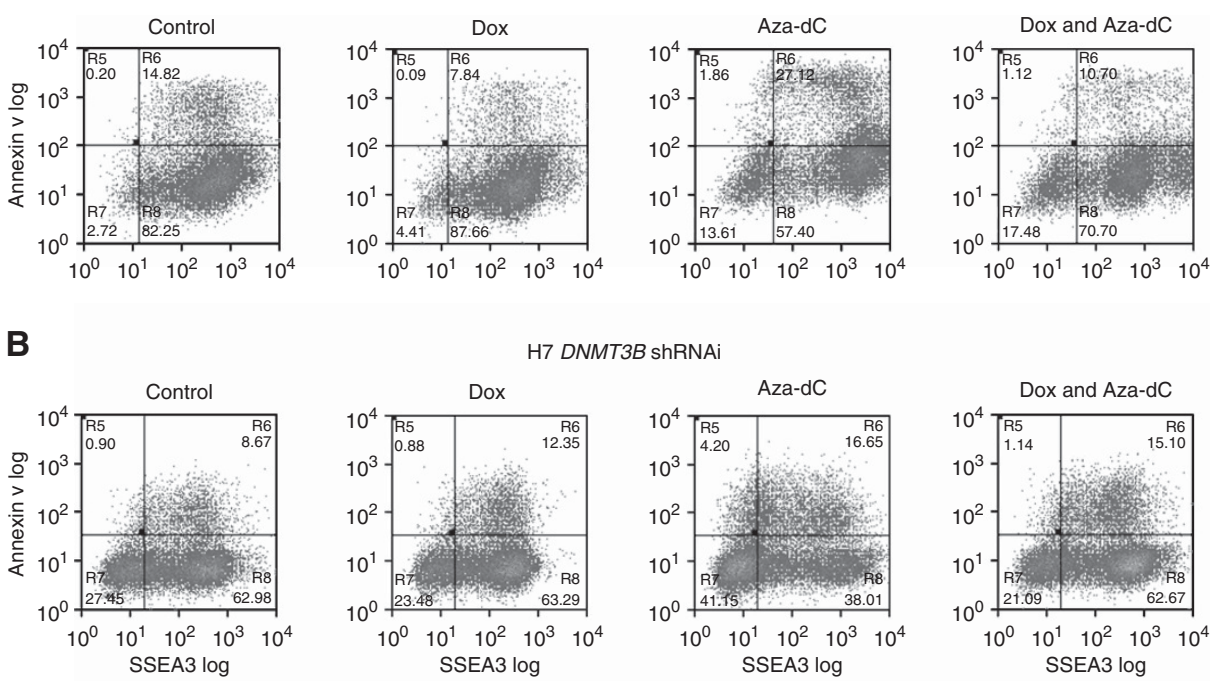

H7 DNMT3B ShRNAi

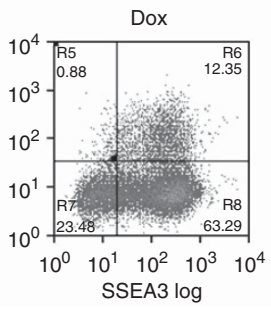

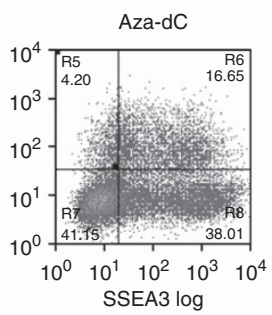

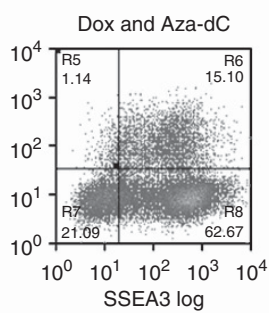

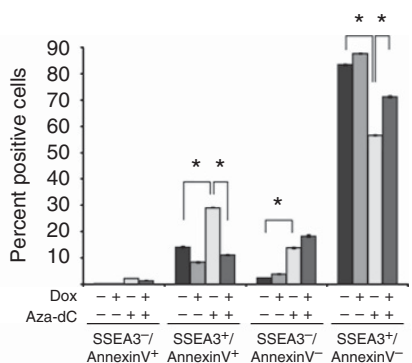

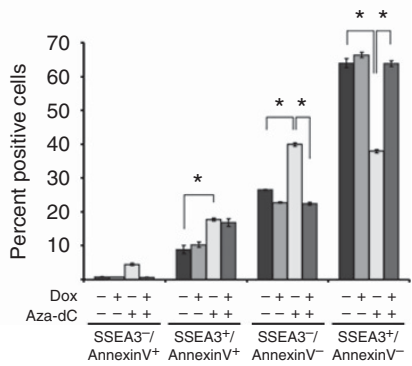

Figure 3. DNMT3B mediates apoptosis of pluripotent stem cells NTERA2 induced by Aza-dC, whereas it mediates differentiation of human ES cells. Flow cytometry analysis of DNMT3B knockdown in (A) NTERA2 and (B) H7 ES cells. Data are represented as mean \pm s.d.; $n=3 ;{ }^{*} P<0.05$.

A

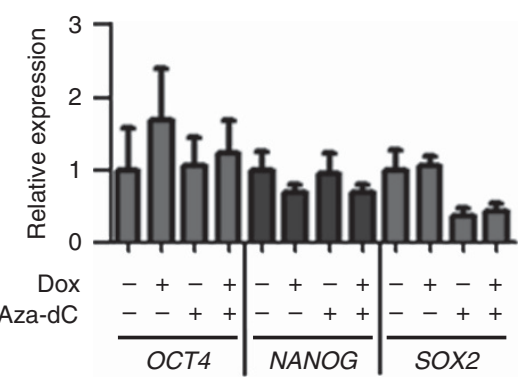

C

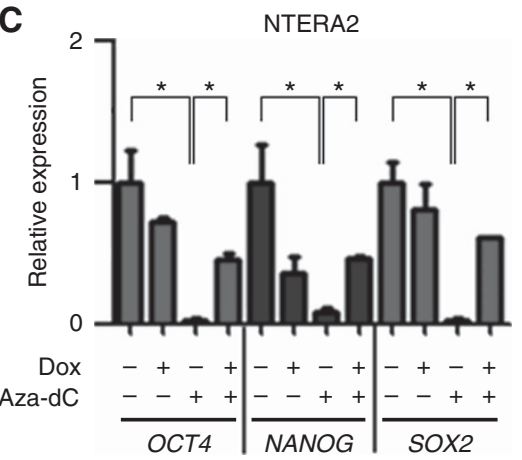

B

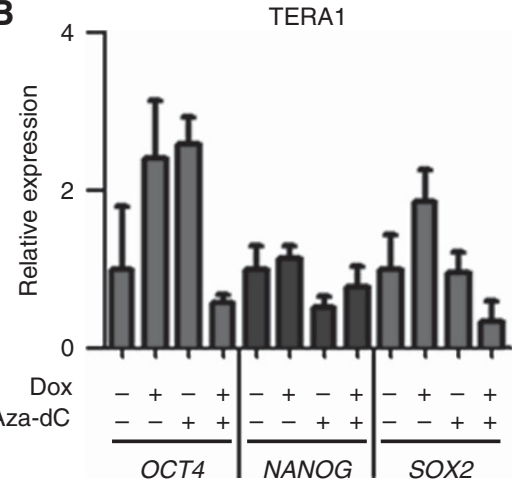

D

$\mathrm{H} 7$

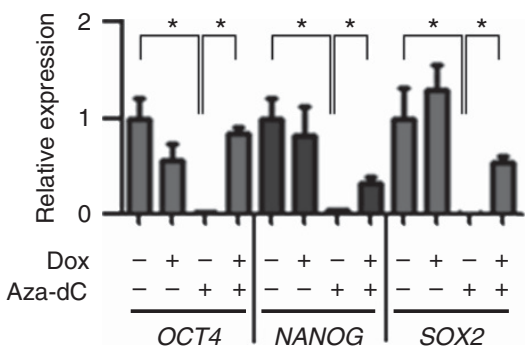

Figure 4. Aza-dC downregulates the expression of stem cell-associated genes in pluripotent stem cells through DNMT3B. Expression of OCT4, NANOG and SOX2 was assessed by RT-qPCR upon treatments by doxycycline (Dox) to knockdown DNMT3B and by Aza-dC in (A) N2102Ep, (B) TERA1, (C) NTERA2 and (D) H7 ES cells. Data are represented as mean \pm s.d.; $n=3 ;{ }^{\star} P<0.05$.

Dose-response experiments were then performed to ascertain whether the sensitivity to Aza-dC is altered in differentiated versus undifferentiated cells. We found that undifferentiated N2102Ep and TERA1 show $\mathrm{IC}_{50}$ at approximately $5 \mathrm{~nm}$, whereas differentiated cells, as induced by Dox treatment to knockdown OCT4, show $\mathrm{IC}_{50}$ at $100 \mathrm{~nm}$ (Figure 5C). This result indicates that the cancer stem cells are more sensitive to Aza-dC than their differentiated derivatives.
Flow cytometric analysis reveals that silencing of OCT4 gave rise to more Annexin $\mathrm{V}^{+}$cells (Figure $2 \mathrm{C}$ and $\mathrm{D}$ ), suggesting that OCT4 might inhibit apoptosis of nullipotent EC cells. We found that expression of DNMT3B was diminished by silencing of OCT4 (Figure 5A) supporting the role of DNMT3B in preventing apoptosis in N2102Ep and TERA1 (Figure 2A and B). Next, upon Aza-dC treatment we compared the level of Annexin $\mathrm{V}^{+}$cells of 

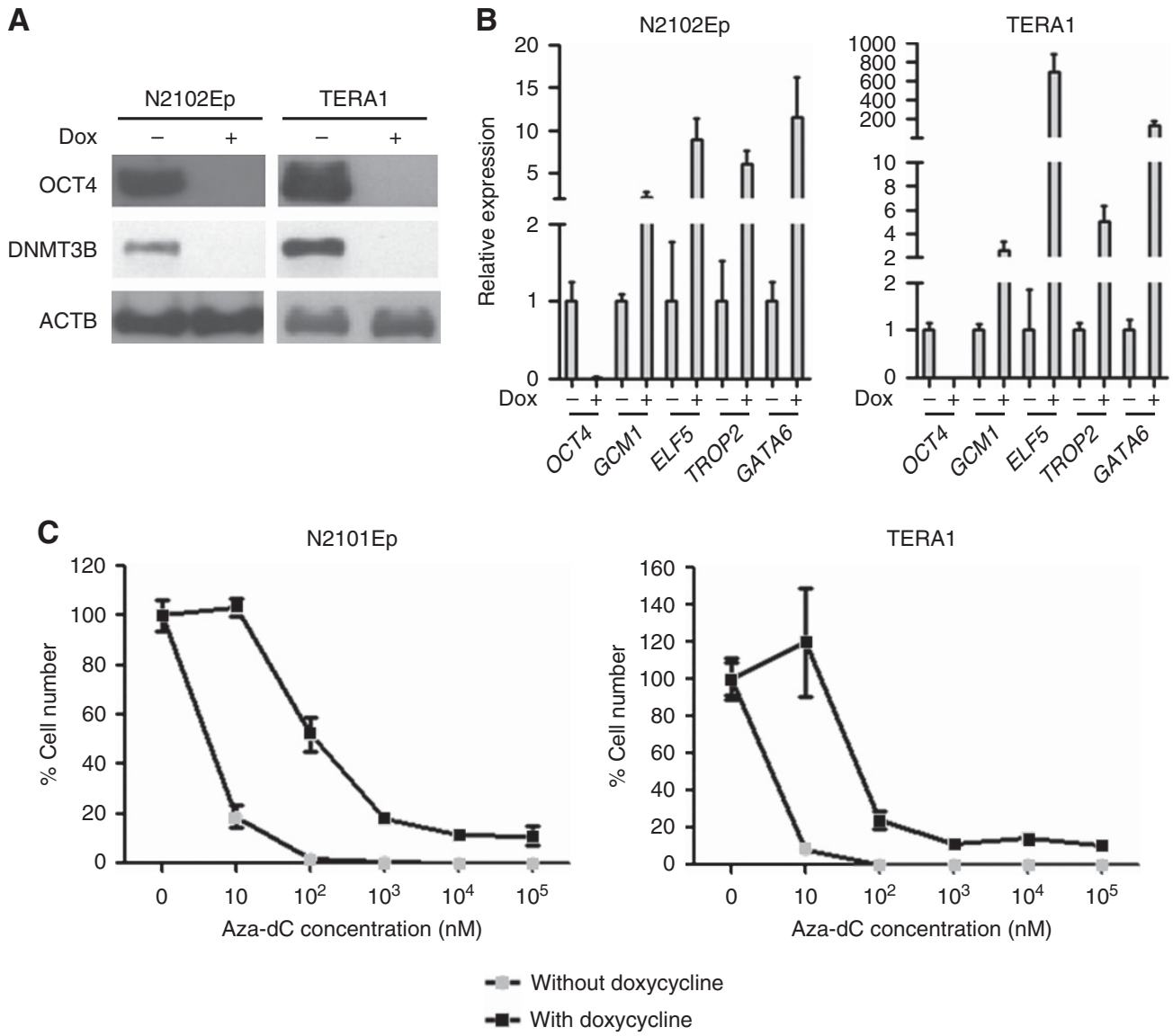

Figure 5. Nullipotent EC cells are more sensitive to Aza-dC than their differentiated cells. (A) Western blotting of N2102Ep and TERA1 clones with OCT4 silencing. Expression of DNMT3B was also downregulated by silencing of OCT4. (B) Expression of differentiation-associated genes was upregulated in OCT4 knockdown EC cells. (C) Dose response of Aza-dC on cell number of EC cells N2102Ep and TERA1 upon OCT4 silencing was determined. Cells were cultured for 7 days with new culture medium containing indicated concentration of Aza-dC replaced every day. Data are represented as mean \pm s.d.; $n=3$.

the stem cells and their OCT4 knockdown differentiated cells to ascertain whether Aza-dC is able to induce apoptosis of the differentiated cancer cells. Although Aza-dC treatment increased the number of Annexin $\mathrm{V}^{+}$cells of EC cells, it failed to do so in the OCT4 knockdown differentiated cancer cells (Figure 2C and D). This result suggests that the apoptotic response induced by Aza-dC is specific to undifferentiated stem cells. Collectively, our results imply that Aza-dC selectively target the stem cell component of teratocarcinoma.

\section{DISCUSSION}

Human teratocarcinoma consists of EC cells, which represent the stem cells of the tumours, and their differentiated cells or teratoma. The majority of human EC cells do not differentiate in culture, therefore they are considered as nullipotent stem cells (Andrews et $a l, 2005)$. DNMT3B is one of the most highly expressed transcripts found in EC cells including N2102Ep and TERA1 (Sperger et al, 2003). We report in the present study that the clonal ability of EC cells is dependent on DNMT3B expression (Figure 1B). DNMT3B is also necessary to prevent apoptosis in EC cells N2102Ep and TERA1, but not in pluripotent NTERA2 and ES cells (Figure 2A and B and Figure $3 \mathrm{~A}$ and B). DNMT3B has been reported as an oncogene in many somatic cancers (Beaulieu et al, 2002; Rhee et al, 2002; Lin et al, 2006; Linhart et al, 2007; Kurita et al, 2010; Sandhu et al, 2011). Our study, therefore, provides evidence that DNMT3B might act as an oncogene in nullipotent EC cells. Similar to our finding, knockdown of DNMT3L, which is another DNA methyltransferase without the catalytic activity, induces two-fold increase of numbers of apoptotic cells in human EC cells (Minami et al, 2010). As DNMT3L interacts with and promotes de novo DNA methylation activity of DNMT3B (Suetake et al, 2004), the role of these two genes might be functionally related in tumorigenesis of teratocarcinoma.

We find that Aza-dC induces apoptosis of nullipotent EC cells (N2102Ep and TERA1) as well as pluripotent EC and ES cells (NTERA2 and H7). Our result supports a recent study, which shows that Aza-dC treatment at the concentration of $10 \mathrm{~nm}$ is enough to attenuate clonal ability and to induce apoptosis of NTERA2 (Beyrouthy et al, 2009). This concentration is approximately 100-1000 fold lower than that used to induce cytotoxicity of somatic cells (Beyrouthy et al, 2009). We show that Aza-dC induces not only apoptosis, but also differentiation of pluripotent NTERA2 and H7 (Figure 3A and B). Our results suggest that the epigenetic drug Aza-dC alters fates of pluripotent stem cells by driving these cells toward differentiation and apoptosis. On the other hand, it induces nullipotent EC cells N2102Ep and TERA1 to undergo only apoptosis but not differentiation.

We show in this study that DNMT3B mediates apoptosis induced by Aza-dC in pluripotent NTERA2 (Figure 3A), whereas it mediates downregulation of SSEA3 expression induced by Aza-dC in pluripotent H7 ES cells (Figure 3B). Nonetheless, DNMT3B has a 
role in Aza-dC-induced downregulation of OCT4, NANOG and SOX2 expression in both NTERA2 and H7 (Figure 4). Our results support previous findings that DNMT3B mediates the effects of Aza-dC to induce apoptosis in mouse ES cells and human pluripotent stem cells NTERA2 (Oka et al, 2005; Beyrouthy et al, 2009; Biswal et al, 2012). In contrast to the pluripotent stem cells, as DNMT3B is important to prevent apoptosis of N2102Ep and TERA1 (Figure 2A and B), knockdown of DNMT3B in the nullipotent N2102Ep and TERA1 might not allow the nullipotent EC cells to encounter the apoptotic effect of Aza-dC. Hence, the sensitivity of Aza-dC toward apoptotic or differentiation responses is not always determined by the expression level of DNMT3B. A mechanism underlying cytotoxicity induced by Aza-dC has been proposed to occur through a covalent trapping of DNA methyltransferases (Juttermann et al, 1994), which then induces DNA mutagenicity (Jackson-Grusby et al, 1997). Given that cell cycle profile is not different in mouse ES cells depleted for DNA methyltransferases (Oka et al, 2005), an incorporation activity of Aza-dC into genomic DNA of the cells might be similar. Therefore, the higher apoptosis induced by Aza-dC treatment in control than in DNMT3B knockdown cells, which was observed only in NTERA2 but not other cell lines, could be explained by the formation of covalent adduct between Aza-dC-DNMT3B at genomic level rather than by difference in cell cycle profile especially in $\mathrm{S}$ phase.

We have previously reported that transient siRNA silencing of OCT4 in nullipotent stem cells including the N2102Ep and TERA1 induced them to differentiate (Matin et al, 2004; Andrews et al, 2005). We show that silencing of OCT4 led to apoptosis of N2102Ep and TERA1 (Figure 2C and D). The increase in apoptotic cells might be due to a reduction of DNMT3B level (Figure 5A), as DNMT3B prevents apoptosis in the nullipotent EC cells (Figure 2A and B). Because somatic cells respond poorly to Aza-dC compared with teratocarcinoma stem cells (Beyrouthy et al, 2009), we induced differentiation of N2102Ep and TERA1 using shRNAiinducible OCT4 knockdown, and found that differentiated OCT4 knockdown cells respond poorly to the epigenetic drug Aza-dC (Figure 2C and D). As Aza-dC is a nucleoside analogue that can be incorporated into DNA during $\mathrm{S}$ phase, the higher cytotoxicity observed in the nullipotent EC cells than in their OCT4 knockdown differentiated cells might be due to a higher cell division rate of the cancer stem cells. Our study, therefore, provides evidence that EC cells but not their differentiated derivatives are sensitive to the epigenetic drug Aza-dC. Therefore, using Aza-dC in clinical trials of patients with teratocarcinoma should be cautious, in which the drug will target only EC cells, but might act poorly against differentiated teratoma tissues. A recent study has also suggested the ability of Aza-dC to target cancer stem-like cells (Tsai et al, 2012). Thus further investigations on the anticancer stem cell property of Aza-dC toward other types of cancers, which are developed from cancer stem cells or cancer initiating cells, will be important to translate the use of this epigenetic drug into clinic.

\section{ACKNOWLEDGEMENTS}

This research was supported by the Thai Commission on Higher Education to PW.

\section{REFERENCES}

Alagaratnam S, Harrison NJ, Bakken AC, Hoff AM, Jones M, Sveen A, Moore H, Andrews P, Lothe RA, Skotheim RI (2012) Transforming pluripotency: an exon-level study of malignancy-specific transcripts in human embryonal carcinoma and embryonic stem cells. Stem Cells Dev 22: $1136-1146$.
Almstrup K, Hoei-Hansen CE, Wirkner U, Blake J, Schwager C, Ansorge W, Nielsen JE, Skakkebaek NE, Rajpert-De Meyts E, Leffers H (2004) Embryonic stem cell-like features of testicular carcinoma in situ revealed by genome-wide gene expression profiling. Cancer Res 64(14): 4736-4743.

Almstrup K, Nielsen JE, Mlynarska O, Jansen MT, Jorgensen A, Skakkebaek NE, Rajpert-De Meyts E (2010) Carcinoma in situ testis displays permissive chromatin modifications similar to immature foetal germ cells. Br J Cancer 103(8): 1269-1276.

Andrews PW (1984) Retinoic acid induces neuronal differentiation of a cloned human embryonal carcinoma cell line in vitro. Dev Biol 103(2): 285-293.

Andrews PW, Bronson DL, Benham F, Strickland S, Knowles BB (1980) A comparative study of eight cell lines derived from human testicular teratocarcinoma. Int J Cancer 26(3): 269-280.

Andrews PW, Damjanov I, Simon D, Banting GS, Carlin C, Dracopoli NC, Fogh J (1984) Pluripotent embryonal carcinoma clones derived from the human teratocarcinoma cell line Tera-2. Differentiation in vivo and in vitro. Lab Invest 50(2): 147-162.

Andrews PW, Matin MM, Bahrami AR, Damjanov I, Gokhale P, Draper JS (2005) Embryonic stem (ES) cells and embryonal carcinoma (EC) cells: opposite sides of the same coin. Biochem Soc Trans 33(Pt 6): 1526-1530.

Beaulieu N, Morin S, Chute IC, Robert MF, Nguyen H, MacLeod AR (2002) An essential role for DNA methyltransferase DNMT3B in cancer cell survival. J Biol Chem 277(31): 28176-28181.

Beyrouthy MJ, Garner KM, Hever MP, Freemantle SJ, Eastman A, Dmitrovsky E, Spinella MJ (2009) High DNA methyltransferase 3B expression mediates 5 -aza-deoxycytidine hypersensitivity in testicular germ cell tumors. Cancer Res 69(24): 9360-9366.

Biswal BK, Beyrouthy MJ, Hever-Jardine MP, Armstrong D, Tomlinson CR, Christensen BC, Marsit CJ, Spinella MJ (2012) Acute hypersensitivity of pluripotent testicular cancer-derived embryonal carcinoma to low-dose 5-aza deoxycytidine is associated with global DNA damage-associated p53 activation, anti-pluripotency and DNA demethylation. PLoS One 7(12): e53003.

Draper JS, Pigott C, Thomson JA, Andrews PW (2002) Surface antigens of human embryonic stem cells: changes upon differentiation in culture. J Anat 200(Pt 3): 249-258.

Jackson-Grusby L, Laird PW, Magge SN, Moeller BJ, Jaenisch R (1997) Mutagenicity of 5-aza-2'-deoxycytidine is mediated by the mammalian DNA methyltransferase. Proc Natl Acad Sci USA 94(9): 4681-4685.

Jones PA, Taylor SM (1980) Cellular differentiation, cytidine analogs and DNA methylation. Cell 20(1): 85-93.

Juttermann R, Li E, Jaenisch R (1994) Toxicity of 5-aza-2'-deoxycytidine to mammalian cells is mediated primarily by covalent trapping of DNA methyltransferase rather than DNA demethylation. Proc Natl Acad Sci USA 91(25): 11797-11801.

Kristensen DM, Sonne SB, Ottesen AM, Perrett RM, Nielsen JE, Almstrup K, Skakkebaek NE, Leffers H, Rajpert-De Meyts E (2008) Origin of pluripotent germ cell tumours: the role of microenvironment during embryonic development. Mol Cell Endocrinol 288(1-2): 111-118.

Kurita S, Higuchi H, Saito Y, Nakamoto N, Takaishi H, Tada S, Saito H, Gores GJ, Hibi T (2010) DNMT1 and DNMT3b silencing sensitizes human hepatoma cells to TRAIL-mediated apoptosis via up-regulation of TRAIL-R2/DR5 and caspase-8. Cancer Sci 101(6): 1431-1439.

Lin H, Yamada Y, Nguyen S, Linhart H, Jackson-Grusby L, Meissner A, Meletis K, Lo G, Jaenisch R (2006) Suppression of intestinal neoplasia by deletion of Dnmt3b. Mol Cell Biol 26(8): 2976-2983.

Linhart HG, Lin H, Yamada Y, Moran E, Steine EJ, Gokhale S, Lo G, Cantu E, Ehrich M, He T, Meissner A, Jaenisch R (2007) Dnmt3b promotes tumorigenesis in vivo by gene-specific de novo methylation and transcriptional silencing. Genes Dev 21(23): 3110-3122.

Matin MM, Walsh JR, Gokhale PJ, Draper JS, Bahrami AR, Morton I, Moore HD, Andrews PW (2004) Specific knockdown of Oct4 and beta2-microglobulin expression by RNA interference in human embryonic stem cells and embryonic carcinoma cells. Stem Cells 22(5): 659-668.

Minami K, Chano T, Kawakami T, Ushida H, Kushima R, Okabe H, Okada Y, Okamoto K (2010) DNMT3L is a novel marker and is essential for the growth of human embryonal carcinoma. Clin Cancer Res 16(10): 2751-2759.

Oka M, Meacham AM, Hamazaki T, Rodic N, Chang LJ, Terada N (2005) De novo DNA methyltransferases Dnmt3a and Dnmt3b primarily mediate the cytotoxic effect of 5-aza-2'-deoxycytidine. Oncogene 24(19): 3091-3099.

Oosterhuis JW, Looijenga LH (2005) Testicular germ-cell tumours in a broader perspective. Nat Rev Cancer 5(3): 210-222. 
Rajpert-de Meyts E, Hoei-Hansen CE (2007) From gonocytes to testicular cancer: the role of impaired gonadal development. Ann N Y Acad Sci 1120: $168-180$.

Rajpert-De Meyts E, Jorgensen N, Brondum-Nielsen K, Muller J, Skakkebaek NE (1998) Developmental arrest of germ cells in the pathogenesis of germ cell neoplasia. Apmis 106(1): 198-204; discussion 204-6.

Rhee I, Bachman KE, Park BH, Jair KW, Yen RW, Schuebel KE, Cui H, Feinberg AP, Lengauer C, Kinzler KW, Baylin SB, Vogelstein B (2002) DNMT1 and DNMT3b cooperate to silence genes in human cancer cells. Nature 416(6880): 552-556.

Sandhu R, Rivenbark AG, Coleman WB (2011) Enhancement of chemotherapeutic efficacy in hypermethylator breast cancer cells through targeted and pharmacologic inhibition of DNMT3b. Breast Cancer Res Treat 131: 385-399.

Shevinsky LH, Knowles BB, Damjanov I, Solter D (1982) Monoclonal antibody to murine embryos defines a stage-specific embryonic antigen expressed on mouse embryos and human teratocarcinoma cells. Cell 30(3): 697-705.

Skakkebaek NE (1972) Possible carcinoma-in-situ of the testis. Lancet 2(7776): 516-517.

Skotheim RI, Lind GE, Monni O, Nesland JM, Abeler VM, Fossa SD, Duale N, Brunborg G, Kallioniemi O, Andrews PW, Lothe RA (2005) Differentiation of human embryonal carcinomas in vitro and in vivo reveals expression profiles relevant to normal development. Cancer Res 65(13): 5588-5598.

Sonne SB, Almstrup K, Dalgaard M, Juncker AS, Edsgard D, Ruban L, Harrison NJ, Schwager C, Abdollahi A, Huber PE, Brunak S, Gjerdrum LM, Moore HD, Andrews PW, Skakkebaek NE, Rajpert-De Meyts E, Leffers H (2009) Analysis of gene expression profiles of microdissected cell populations indicates that testicular carcinoma in situ is an arrested gonocyte. Cancer Res 69(12): 5241-5250.

Sperger JM, Chen X, Draper JS, Antosiewicz JE, Chon CH, Jones SB, Brooks JD, Andrews PW, Brown PO, Thomson JA (2003) Gene expression patterns in human embryonic stem cells and human pluripotent germ cell tumors. Proc Natl Acad Sci USA 100(23): 13350-13355.

Suetake I, Shinozaki F, Miyagawa J, Takeshima H, Tajima S (2004) DNMT3L stimulates the DNA methylation activity of Dnmt3a and Dnmt3b through a direct interaction. J Biol Chem 279(26): 27816-27823.

Taylor SM, Jones PA (1979) Multiple new phenotypes induced in 10T1/2 and 3T3 cells treated with 5-azacytidine. Cell 17(4): 771-779.

Thomson JA, Itskovitz-Eldor J, Shapiro SS, Waknitz MA, Swiergiel JJ, Marshall VS, Jones JM (1998) Embryonic stem cell lines derived from human blastocysts. Science 282(5391): 1145-1147.

Tsai HC, Li H, Van Neste L, Cai Y, Robert C, Rassool FV, Shin JJ, Harbom KM, Beaty R, Pappou E, Harris J, Yen RW, Ahuja N, Brock MV, Stearns V, Feller-Kopman D, Yarmus LB, Lin YC, Welm AL, Issa JP, Minn I, Matsui W, Jang YY, Sharkis SJ, Baylin SB, Zahnow CA (2012) Transient low doses of DNA-demethylating agents exert durable antitumor effects on hematological and epithelial tumor cells. Cancer cell 21(3): 430-446.

Wongtrakoongate P, Li J, Andrews PW (2014) DNMT3B inhibits the re-expression of genes associated with induced pluripotency. Exp Cell Res 321(2): 231-239.

Yang X, Lay F, Han H, Jones PA (2010) Targeting DNA methylation for epigenetic therapy. Trends Pharmacol Sci 31(11): 536-546.

Zafarana G, Avery SR, Avery K, Moore HD, Andrews PW (2009) Specific knockdown of OCT4 in human embryonic stem cells by inducible short hairpin RNA interference. Stem Cells 27(4): 776-782.

This work is published under the standard license to publish agreement. After 12 months the work will become freely available and the license terms will switch to a Creative Commons AttributionNonCommercial-Share Alike 3.0 Unported License. 